Research Article

\title{
Optimal Design of Multiband Microstrip Antennas by Self-Renewing Fitness Estimation of Particle Swarm Optimization Algorithm
}

\author{
Xiaohong Fan $(\mathbb{D}$, Yubo Tian $(\mathbb{D}$, and Yi Zhao \\ School of Electronics and Information, Jiangsu University of Science and Technology, Zhenjiang 212003, Jiangsu, China \\ Correspondence should be addressed to Xiaohong Fan; 18362895409@163.com
}

Received 28 January 2019; Revised 16 April 2019; Accepted 19 August 2019; Published 12 September 2019

Academic Editor: Xiulong Bao

Copyright ( 2019 Xiaohong Fan et al. This is an open access article distributed under the Creative Commons Attribution License, which permits unrestricted use, distribution, and reproduction in any medium, provided the original work is properly cited.

\begin{abstract}
In order to reduce the time of designing microstrip antenna, this paper proposes a self-renewing fitness estimation of particle swarm optimization algorithm (SFEPSO) to improve the design efficiency. Firstly, a fitness predictive model of the particles is constructed according to the evolution formula of particle swarm optimization (PSO). From the third generation of the algorithm, the fitness of particles is given by the prediction model instead of the full-wave electromagnetic simulation. Aiming to keep the right direction of evolution, the prediction model will be proofed every $N$ generation during the optimization process. If the prediction model accuracy is lower than the threshold, it will be updated and then continue iterating until the particles satisfy the demands. Compared with the traditional optimization method, this method greatly reduces the evaluation time and improves the design efficiency. The method is validated by the optimized design of E-type dual-frequency microstrip antenna and WLAN/ WiMAX multiband antenna. The optimized results show that the purpose of rapid optimization can be achieved while ensuring the design accuracy.
\end{abstract}

\section{Introduction}

In the field of wireless communication, antennas are not only the front-end devices for transmitting and receiving electromagnetic waves but also the conversion devices between high-frequency current energy and electromagnetic wave energy. Among kinds of antennas, microstrip antennas (MSAs) are widely used in various communication systems due to their advantages of light, small, low profile, and easy dual-frequency or multifrequency [1]. When designing the MSAs, the methods of computer-assisted design (CAD) and computer numerical analysis have been developed and applied. Now, the most commonly used full-wave electromagnetic simulation softwares are HFSS, CST, and IE3D. Global optimization algorithm calling full-wave electromagnetic simulation software can optimally design antennas accurately. However, this method needs to call the full-wave electromagnetic simulation software again and again for evaluating the fitness of individuals. The calculation cost is too high, and the design efficiency is very low.
In recent years, the machine-learning method has greatly saved the time when optimizing design MSAs after being applied in the field of electromagnetism [2-4]. At present, the most commonly used methods are artificial neural network (ANN) [5-8], support vector machine (SVM) $[9,10]$, and Gaussian process (GP) [11]. These methods replace the true fitness calculation by constructing a prediction model, which shortens the time in entire optimization process by reducing the number of evaluations of the fitness calculation. These have been successfully applied in antennas design. Neog et al. calculated the antenna pattern through the ANN and combined the genetic algorithm (GA) to analyze the resonant frequency of the antenna [12]. Chen et al. proposed a GPR-based knowledge neural network in [13] and applied it in MSAs. Roy et al. used the SVM to calculate the performance parameters such as the resonant frequency, gain, and directivity of the slotted MSA and achieved good agreement between the predicted results and the measured results [14]. Sun et al. [15] proposed a SVM combined with a 
hybrid kernel function for accurately modeling the resonant frequency of a compact MSA. Jacobs and Villier proposed GP to model ultrawideband and dual-frequency coplanar waveguide-fed slot antennas [16, 17]. Jacobs proposed a design method for resonant frequency modeling of dual-frequency MSAs based on GP [18]. In general, these prediction models usually approximate the function by learning a large number of samples. The correctness of the model has a great relationship with the sample selection. And as the dimension increases, the difficulty of building a prediction model will increase. For ANN, the training requires a large number of samples. When the data are insufficient, the generalization ability of the trained ANN is not excellent. When the number of observation samples is large, the working efficiency will be significantly reduced, and there is no general solution to the nonlinear problem for SVM because different problems need to choose different kernel functions. If the selected kernel function is not appropriate, it will bring big difficulties for SVM modeling. Comparing to ANN and SVM, GP has the advantages of being suitable for dealing with nonlinear problems and the output with probability meaning, but it has the disadvantages of large computational complexity because of its nonparametric property. At the same time, the noise must obey Gaussian distribution. When dealing with more complex problems, it is difficult to find suitable kernel functions and optimal hyperparameters of GP and SVM.

The fitness inheritance method is another approach to predict the fitness; that is, the fitness of the children inherits that of the parents in a certain way. Different from the sample prediction model, the inherited prediction model can save a lot of sample acquisition time and also avoid errors of prediction caused by improper sampling methods. Smith et al. [19] used the inherited fitness instead of true fitness in the evolution of GA, and the fitness of some individuals in the population is directly assigned by the average value of the fitness of their parents. Salami and Hendtlass proposed a fast evolutionary algorithm in which the fitness of the offspring is directly assigned by the weighted average of the fitness of the parents [20]. However, GA has the disadvantages of low search efficiency and strong parameter dependence. In recent years, the idea of constructing a fitness prediction model by particle swarm optimization (PSO) algorithm has been proposed. The PSO algorithm has the advantages of not requiring the function continuous or differential, and the number of parameters to be adjusted is small. Margarita et al. introduced the concept of fitness inheritance into the multiobjective PSO [21]. Cui et al. proposed a fast PSO algorithm based on the change of the velocity and position of particles. Only when the reliability of fitness of the particle is below the threshold, the true fitness function needs to be calculated [22]. In order to avoid local convergence, the author has adopted a new strategy; that is, the fitness of the estimated individual is determined by a weighted combination, and the weight of each parent is proportional to the distance between the estimated particle and its parent [23]. Sun et al. proposed a fitness prediction model according to the distance among particles of the same generation; that is, the fitness of any generation of some particles are known, then the fitness of other particle in the evolution can be predicted [24]. Xiao et al. proposed a PSO algorithm based on adaptive penalty function, which makes full use of the information of feasible and infeasible solutions of all adjacent constraint boundaries. The algorithm can perform deep search on the problem solution space until it gets the optimal solution [25]. Lu et al. proposed a PSO algorithm based on affinity propagation clustering, which significantly reduces the computational complexity of the objective function [26]. The fitness inheritance method based on the PSO algorithm can not only avoid the time consumption of sampling but also maintain the excellent performance of the algorithm. According to the evolutionary formula of the PSO algorithm, this paper constructs a self-renewing prediction model for optimal design of MSAs.

The rest of the paper is structured as follows. Section 2 introduces the basic principles of PSO algorithm, fitness estimation method based on PSO algorithm, and Gaussian mutations (FEPSO). Section 3 introduces the implementation of the self-renewing fitness prediction method based on PSO algorithm (SFEPSO). Section 4 presents the application of SFEPSO in E-type dual-frequency MSA. Section 5 presents the application of SFEPSO in WLAN/ WiMAX multiband MSA. Finally, summaries are provided in Section 6.

\section{Related Techniques}

2.1. Particle Swarm Optimization. PSO algorithm is a typical swarm intelligence optimization algorithm that simulates bird swarm in search of food processes [27]. The theory is that collaboration among the particles generates group intelligence to guide search. PSO considers each individual as a particle without weight and volume in space and flies at a certain speed in the search space with reference to the flight experience of the group and the flight experience of the particle itself. In the algorithm, the state vector of each particle usually contains the position and velocity. At the beginning of the search, the state of particles is given randomly within the search range. During the search, there are two important pieces of information that be retained; one is the best location named pbest for each particle, and the other is the best location named gbest for the entire population. The best location is measured by fitness function. Each particle is driven toward its best location and the optimal location of the population.

The PSO can be described in mathematical language. Assuming that the particles search space is $n$-dimension, and the entire particle swarm $x=\left(x_{1}, x_{2}, \cdots, x_{m}\right)^{T}$ contains $m$ particles. The location of the particle $i$ is $x_{i}=\left(x_{i, 1}, x_{i, 2}, \cdots, x_{i, n}\right)^{T}$, and the particle velocity is $v_{i}=\left(v_{i, 1}, v_{i, 2}, \cdots, v_{i, n}\right)^{T}$. When particles find the best individual locations and the global best location, we can use equations (1) and (2) to update their velocity and positions: 


$$
\begin{aligned}
v_{i, d}^{(k+1)}= & \omega v_{i, d}^{(k)}+c_{1} \operatorname{rand}()\left(\text { pbest }_{i, d}^{(k)}-x_{i, d}^{k}\right) \\
& +c_{2} \operatorname{rand}()\left(\text { gbest }_{d}^{(k)}-x_{i, d}^{(k)}\right), \\
x_{i, d}^{(k+1)}= & x_{i, d}^{(k)}+v_{i, d}^{(k+1)},
\end{aligned}
$$

where $\omega$ is the inertia weight factor; $c_{1}$ and $c_{2}$ are the accelerating constants; rand () is a random number between $(0,1)$; $v_{i, d}^{(k)}$ and $x_{i, d}^{(k)}$ are, respectively, the velocity and positions of the $d$ th dimension of particle $i$ in the $k$ iteration; pbest $_{i, d}^{(k)}$ is the $d$ th dimension of the best individual position of particle $i$; and gbest $_{d}^{(k)}$ is the $d$ th dimension of the best position of all particles.

2.2. Fitness Estimation Method Based on PSO (FEPSO). For particle $i$ in the population, the PSO velocity update formula (1) is substituted into the position update formula (2), and we have

$$
\begin{aligned}
x_{i, d}^{(k+1)}= & x_{i, d}^{(k)}+\omega v_{i, d}^{(k)}+c_{1} \operatorname{rand}()\left(\text { pbest }_{i, d}^{(k)}-x_{i, d}^{k}\right) \\
& +c_{2} \operatorname{rand}()\left(\operatorname{gbest}_{d}^{(k)}-x_{i, d}^{(k)}\right) .
\end{aligned}
$$

From (2), we know that

$$
x_{i, d}^{(k)}=x_{i, d}^{(k-1)}+v_{i, d}^{(k)} \text {. }
$$

Thus,

$$
v_{i, d}^{(k)}=x_{i, d}^{(k)}-x_{i, d}^{(k-1)}
$$

Substituting (5) into (3), after rearrangement, it becomes

$$
\begin{aligned}
x_{i, d}^{(k+1)}= & \left(1+\omega-c_{1} \operatorname{rand}()-c_{2} \operatorname{rand}()\right) x_{i, d}^{(k)}-\omega x_{i, d}^{(k-1)} \\
& +c_{1} \operatorname{rand}() \text { pbest }_{i, d}^{(k)}+c_{2} \text { rand }() \text { gbest }_{d}^{(k)} .
\end{aligned}
$$

Formula (6) is the position update formula of particles in FEPSO. We can find that the $(k+1)$ th generation position $x_{i}^{(k+1)}$ of particle $i$ can be obtained by linear combination of $x_{i}^{(k)}$, $x_{i}^{(k-1)}$, pbest $_{i}^{(k)}$, and gbest ${ }^{(k)}$, which means the $(k+1)$ th generation fitness $f\left(x_{i}^{(k+1)}\right)$ of particle $i$ can be obtained by these four fitnesses linearly weighted. The $f\left(x_{i}^{(k+1)}\right)$ can be calculated as follows:

$$
\begin{aligned}
f\left(x_{i}^{(k+1)}\right)= & \frac{1}{\alpha}\left(\frac{1}{d_{i}^{i(k)}} f\left(x_{i}^{(k)}\right)+\frac{1}{d_{i}^{i(k-1)}} f\left(x_{i}^{(k-1)}\right)\right. \\
& \left.+\frac{1}{d_{i}^{p(k)}} f\left(\text { pbest }_{i}^{(k)}\right)+\frac{1}{d_{i}^{g(k)}} f\left(\text { gbest }^{(k)}\right)\right), \\
\alpha= & \frac{1}{d_{i}^{i(k)}}+\frac{1}{d_{i}^{i(k-1)}}+\frac{1}{d_{i}^{p(k)}}+\frac{1}{d_{i}^{g(k)}},
\end{aligned}
$$

where $f\left(x_{i}^{(k-1)}\right), f\left(x_{i}^{(k)}\right)$, and $f\left(x_{i}^{(k+1)}\right)$, respectively, denote the fitness of the $(k-1)$ th, $k$ th, and $(k+1)$ th generation particles $i$, and $d_{i}^{i(k)}, d_{i}^{i(k-1)}, d_{i}^{p(k)}$, and $d_{i}^{g(k)}$, respectively, denote the distances from $x_{i}^{(k+1)}$ to $x_{i}^{(k)}$, $x_{i}^{(k-1)}{\text {, } \text { best }_{i}^{(k)} \text {, and gbest }}^{(k)}$.
2.3. Gaussian Variation. In the last stage of iteration, the PSO algorithm is easy to fall into local optimum. This paper uses the Gaussian variation operator to update the global optimal particle, thus enhancing the particle search ability. Gaussian variation is that a Gaussian variation matrix with a mean of 0 and a standard deviation of 1 is generated by a Gaussian distribution function and the obtained results multiply each dimension of the original particle as an update step. Since the peak of the Gaussian distribution curve is located at the position of the mean value, the Gaussian mutation will focus on the local area near the original particle and the convergence ability of the algorithm is improved. The Gaussian variation formula is given by

$$
\begin{aligned}
& \text { stepsize }=\operatorname{normrnd}(0,1, D), \\
& x_{i}^{(k)} \longleftarrow x_{i}^{(k)}+\text { stepsize } \oplus x_{i}^{(k)},
\end{aligned}
$$

where stepsize is the update step, normrnd $(0,1, D)$ is the Gaussian distribution function, and $D$ is the dimension. $x_{i}^{(k)}$ is the position of $k$ th generation of particle $i$, “ $\oplus$ " represents matrix multiplication, and “—” represents the assignment symbol which assigns $x_{i}^{(k)}+\operatorname{stepsize} \oplus x_{i}^{(k)}$ to $x_{i}^{(k)}$.

\section{Self-Renewing Fitness Prediction Method Based on PSO Algorithm (SFEPSO)}

When optimizing the MSAs, first a set of particles in the search range are randomly generated as the initial population of PSO algorithm. The fitness functions of firstgeneration particles are calculated by HFSS. After updating the velocity and position, the fitness functions of secondgeneration particles are also calculated by HFSS. From the third generation, the fitness functions of the particles are predicted according to equation (7) in which parameters are, respectively, the position information and fitness of the firstand second-generation particles. Similarly, the fitness of the $N$ th generation particles can be obtained from the position information and the fitness of the $(N-1)$ th and the $(N-2)$ th generation ones. In order to ensure the accuracy of the predicted results, the model is proofed every $j$ generations in the optimization process, where $j$ is a constant. If the value of $j$ is too large, the accuracy of the prediction model prediction will be reduced. If the value of $j$ is too small, although the prediction accuracy is high, the time cost will be large. In general, when the $j$ is in $(5,10)$, the predicted fitness can be accurate while it can save optimization time. If the prediction model accuracy is lower than threshold, the prediction model needs to be updated, and then it continues iterating until the particles satisfy the demand. In this paper, the value of $j$ is 8 , and the threshold is 1.3 times of average absolute error (ABE) between predicted and simulated values of HFSS. In [28], we also proposed a fitness estimation-based PSO algorithm. However, sometimes the method was not robust because we did not check the predicted accuracy when evolution. The flow chart of the proposed SFEPSO algorithm in this paper is shown in Figure 1. 


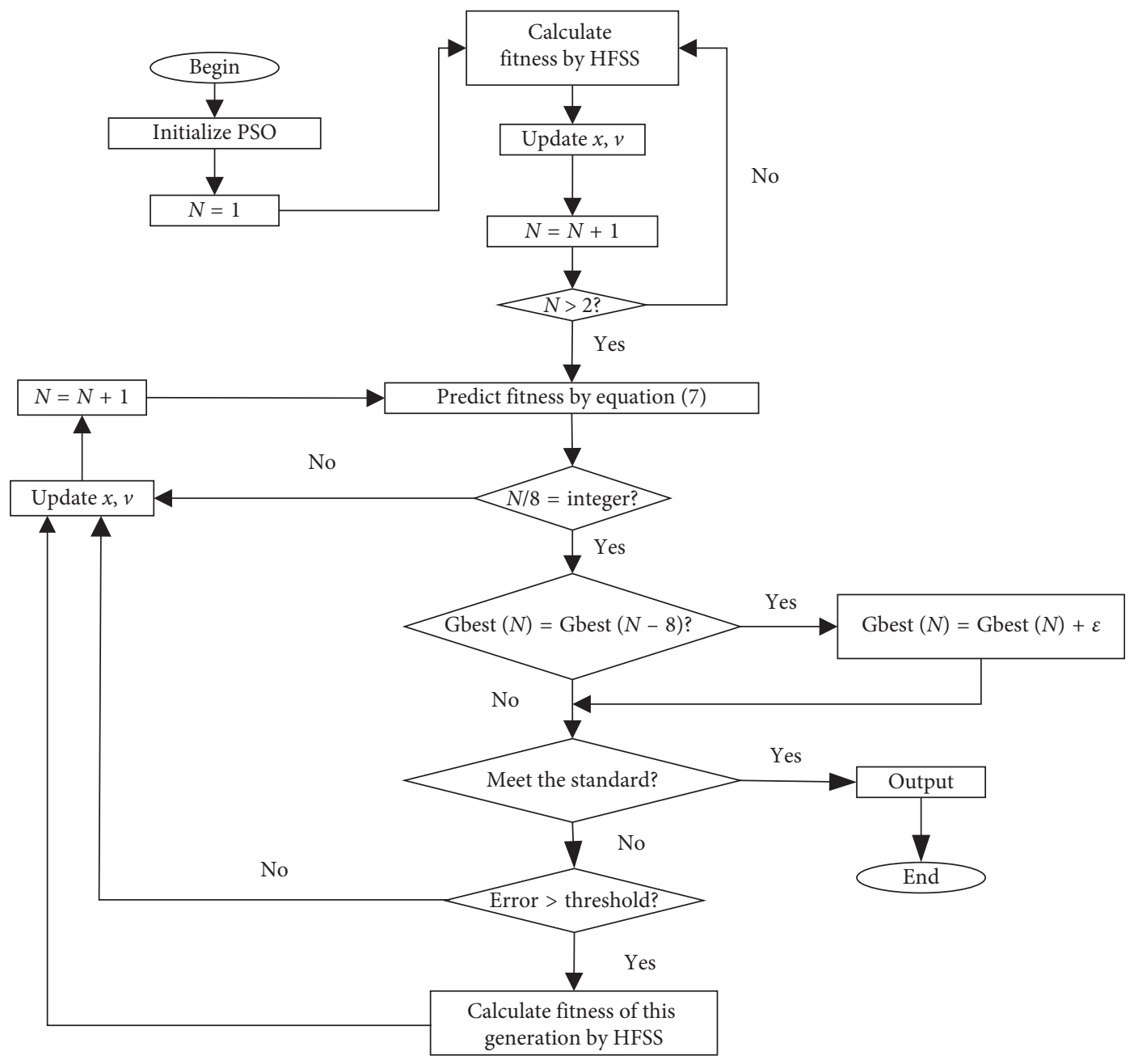

FIgURE 1: Flow chart of SFEPSO algorithm.

The main steps of optimal design of MSAs by the SFEPSO method are as follows:

(1) The antenna to be optimized is modeled in HFSS.

(2) Initialization of PSO algorithm, including population size, inertia weight, cognitive coefficient, and social coefficient.

(3) For the first two generations, calculate the fitness of each particle using HFSS.

(4) According to the particles position and fitness of the first two generations, predict the fitness of the next generation particles by equation (7).

(5) Repeat step 4. When 8 times iteration is reached, we will judge whether the position of the gbest particle at this time is the same as that of the last 8 generations or not. If it is different, go to step 6; otherwise, add a Gaussian variation operator to the gbest of this generation, and then go to step 6 .

(6) Call HFSS to judge whether the gbest has reached the design demand. If reached, the algorithm stops. If not, proceed to step 7 .
(7) Judged whether the error between the fitness calculated by HFSS and the predicted one is less than the threshold or not. If the error is less than the threshold, return to step 5. If not, calculate the fitness of all particles of this generation by HFSS. At the following prediction, we use equation (7) in which $k$ and $(k-1)$ represent, respectively, the generation and the last generation whose fitness is computed with HFSS. After that, return to step 5.

\section{E-Shaped Dual-Frequency MSA}

MSAs have advantages of small size and light weight. This study introduces an E-shaped dual-frequency MSA with 1.9 GHz and $2.4 \mathrm{GHz}$, which is shown in Figure 2. The patch is located at the center of ground, and the dielectric material is air. $G_{L}, G_{W}$, and $H$ are the length, width of ground, and height from patch to ground. $L$ and $W$ are the length and width of the patch. The two slots are with the same size, and $L_{S}$ and $W_{S}$ are the length and width of the slot. $P_{S}$ is the distance from the centerline of the slot to the centerline of the patch. $X_{0}$ is the position of the feed point. The 


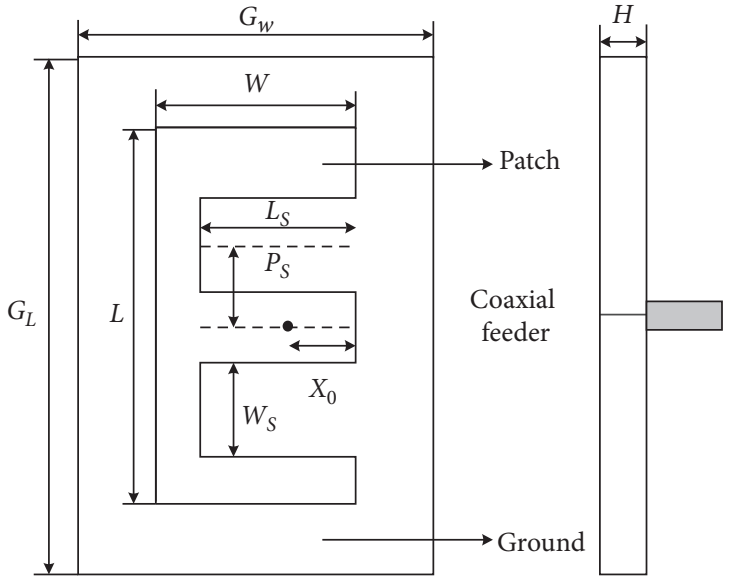

(a)

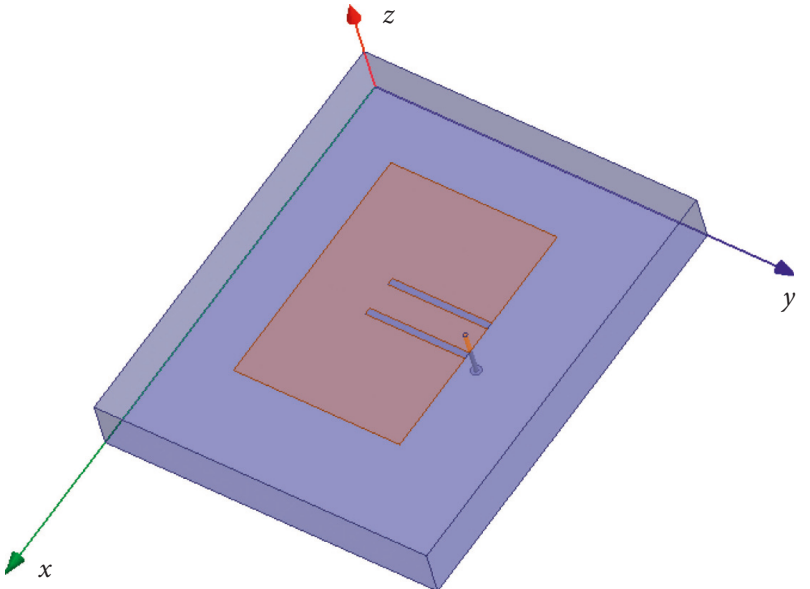

(b)

FIGURE 2: E-shaped dual-frequency MSA. (a) Structure of the E-shaped dual-frequency MSA. (b) E-shaped dual-frequency MSA model in HFSS.

three-dimensional model of the antenna in HFSS is shown in Figure 2(b).

The antenna parameter $v=\left[L_{S} W_{S} P_{S} X_{0}\right]$ is selected in this paper to be the optimized variables where the range of $L_{S}$ is from $30 \mathrm{~mm}$ to $50 \mathrm{~mm}, W_{S}$ from $2 \mathrm{~mm}$ to $10 \mathrm{~mm}, P_{S}$ from $5 \mathrm{~mm}$ to $15 \mathrm{~mm}$, and $X_{0}$ from $4 \mathrm{~mm}$ to $12 \mathrm{~mm}$. Other parameters are fixed and listed in Table 1.

The fitness function is

$$
y_{\max }=\left|\max \left(y_{@ 1.9 \mathrm{GHz}}, y_{@ 2.4 \mathrm{GHz}}\right)\right| \text {, }
$$

where $y_{@ 1.9 \mathrm{GHz}}$ and $y_{@ 2.4 \mathrm{GHz}}$ are $\left|S_{11}\right|$ at $1.9 \mathrm{GHz}$ and $2.4 \mathrm{GHz}$ and $y_{\max }$ is the absolute value of maximum. The bigger the $y_{\max }$, the better the result. In optimization process, the step size of frequency is $0.01 \mathrm{GHz}$.

In the optimization process of the proposed algorithm, the number of particles is 20 and the maximum iterations number is 200. The acceleration constants $c_{1}=c_{2}=2$, and the inertia weight $\omega=1$. The total optimization time is 2.25 hours, and the prediction model is updated 5 times during the optimization. Compared with traditional PSO algorithm calling HFSS software, the efficiency of SFEPSO algorithm is improved by $96.5 \%$. The optimal size is $v=\left[\begin{array}{llll}46.6087 & 10 & 9.04469 & 7.7518\end{array}\right] \mathrm{mm}$, and the $\left|S_{11}\right|$ that satisfies the design specifications is shown in Figure 3.

\section{WLAN/WiMAX Multiband MSA}

The wireless local area network (WLAN) has good flexibility and mobility [29]. Users can access the network anytime and anywhere. Its working frequency is $2.45 \mathrm{GHz}$ (2.4$2.484 \mathrm{GHz}), \quad 5.25 \mathrm{GHz} \quad(5.15-5.35 \mathrm{GHz})$, and $\quad 5.8 \mathrm{GHz}$ $(5.725-5.825 \mathrm{GHz})$. But its coverage is too small. Worldwide Interoperability for Microwave Access (WiMAX) works at $2.5 \mathrm{GHz} \quad(2.5-2.69 \mathrm{GHz}), \quad 3.55 \mathrm{GHz}(3.4-3.69 \mathrm{GHz})$, and $5.5 \mathrm{GHz}(5.25-5.85 \mathrm{GHz})$ frequencies [30]. It has advantages of large coverage and user rapid movement without causing quality degradation. WLAN and WiMAX have gradually replaced older wired computer local area network.
TABLE 1: Fixed parameters of the E-shaped dual-frequency MSA.

\begin{tabular}{lc}
\hline Name & Value $(\mathrm{mm})$ \\
\hline$G_{L}$ & 120 \\
$G_{W}$ & 100 \\
$H$ & 15 \\
$L$ & 70 \\
$W$ & 50 \\
\hline
\end{tabular}

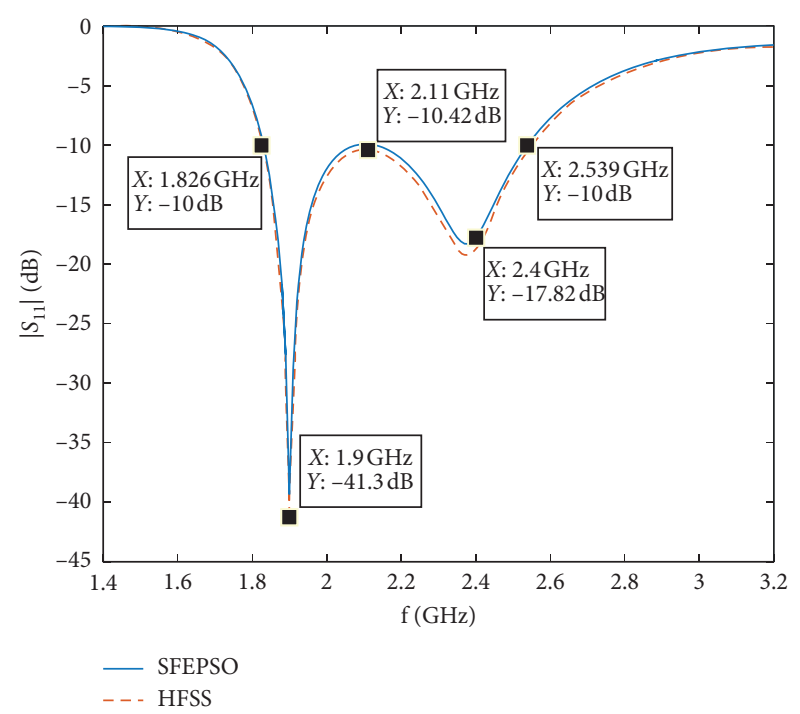

FIGURE 3: Results of the optimized E-shaped dual-frequency MSA.

If we combine WLAN and WiMAX, we can make up for their deficiencies and take advantage of them. Therefore, it is practical to study WLAN/WiMAX multiband MSA. The WLAN/WiMAX multiband antenna designed in this study is shown in Figure 4. The relative dielectric constant of dielectric substrate is 4.4 , and $L_{1}, W_{1}$, and $H$ are, respectively, the length, width, and height of the dielectric substrate. Length and width of the ground plate are $L_{9}$ and $W_{5}$. The 


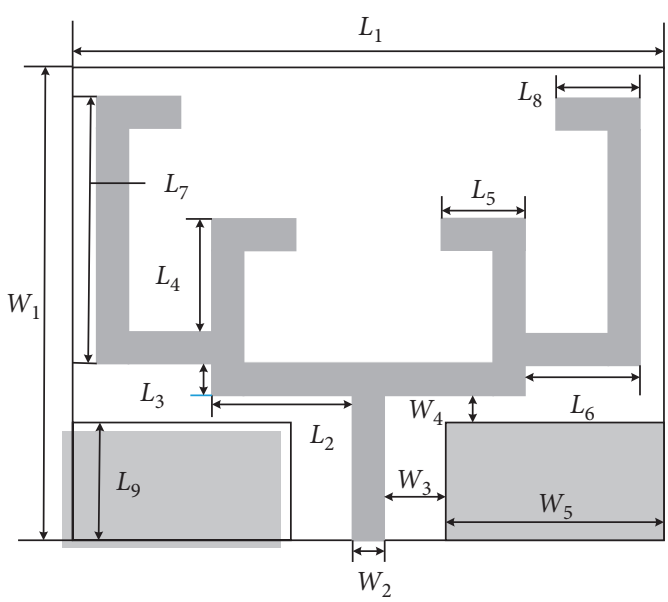

(a)

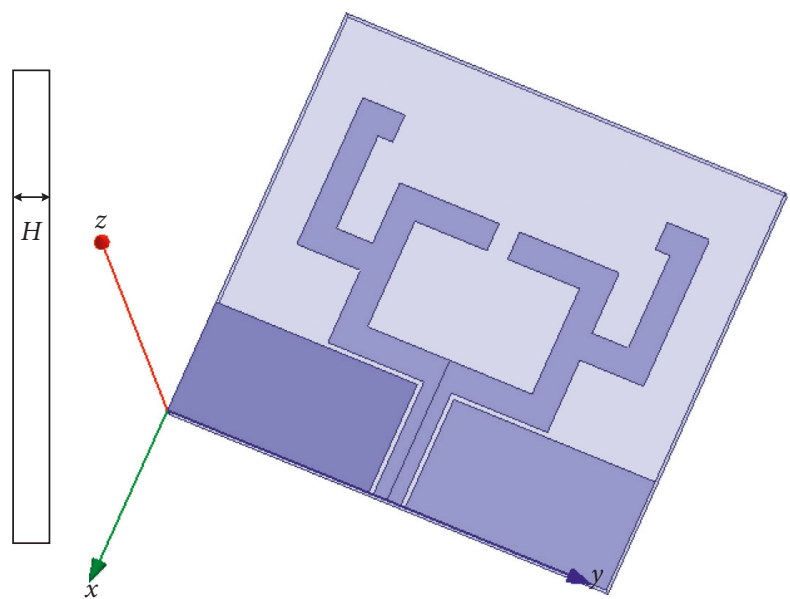

(b)

FIGURE 4: WLAN/WiMAX multiband MSA. (a) Structure of WLAN/WiMAX multiband antenna. (b) WLAN/WiMAX multiband antenna model in HFSS.

antenna is fed by coplanar waveguide (CPW), and the center line width is $W_{2}$; the distance between the strip line and the ground plate is $W_{3}$. The lengths of the antenna branches are $L_{2}, L_{3}, L_{4}, L_{5}, L_{6}, L_{7}$, and $L_{8}$.

The working frequencies of the antenna are $2.4 \mathrm{GHz}$, $3.4 \mathrm{GHz}$, and $5.6 \mathrm{GHz}$, and $-10 \mathrm{~dB}$ bandwidth covering WLAN frequency range and WiMAX frequency band which is $2.4-2.484 \mathrm{GHz}, \quad 5.15-5.35 \mathrm{GHz}, 5.725-5.825 \mathrm{GHz}$, and 2.5-2.69GHZ, 3.4-3.69 GHz, 5.25-5.85 GHz. The parameter $v=\left[L_{4} L_{5} L_{6} L_{7} L_{8}\right]$ is selected to be optimized variables where the range of $L_{4}$ is from $3.5 \mathrm{~mm}$ to $9 \mathrm{~mm}, L_{5}$ from $3.5 \mathrm{~mm}$ to $12 \mathrm{~mm}, L_{6}$ from $3.5 \mathrm{~mm}$ to $12 \mathrm{~mm}, L_{7}$ from $15 \mathrm{~mm}$ to $22 \mathrm{~mm}$, and $L_{8}$ from $3.5 \mathrm{~mm}$ to $6 \mathrm{~mm}$. Other fixed dimensions are listed in Table 2.

The fitness function is given by

$$
y=y_{1} \& \& y_{2} \& \& y_{3}
$$

where

$$
\begin{aligned}
y_{1}= & || y_{@ 2.2 \mathrm{GHz}}|-10|<0.5 \& \& \| y_{@ 2.7 \mathrm{GHz}} \mid \\
& -10|<0.5 \& \&| \max \left(y_{@ 2.45 \mathrm{GHz}}, y_{@ 2.55 \mathrm{GHz}}\right) \mid>10, \\
y_{2}= & || y_{@ 3.4 \mathrm{GHz}}|-10|<0.5 \& \&|| y_{@ 3.8 \mathrm{GHz}} \mid \\
& -10|<0.5 \& \&| y_{@ 3.55 \mathrm{GHz}} \mid>10, \\
y_{3}= & || y_{@ 5.1 \mathrm{GHz}}|-10|<0.5 \& \&|| y_{@ 5.9 \mathrm{GHz}}|-10| \\
& <0.5 \& \&\left|\max \left(y_{@ 5.25 \mathrm{GHz}}, y_{@ 5.4 \mathrm{GHz}}, y_{@ 5.5 \mathrm{GHz}}\right)\right|>10,
\end{aligned}
$$

where $y_{@ 2.2 \mathrm{GHz}}$ is the $\left|S_{11}\right|$ at $2.2 \mathrm{GHz}$ and the expressions of other frequencies in (13)-(15) are the same as $y_{@ 2.2 \mathrm{GHz}}$.

The maximum iterations number is 500 . The other parameters of the proposed method for the multiband MSA are same with the E-shaped MSA. The total optimization time is 21.55 hours, and the prediction model is updated 22 times during the optimization process. Compared with traditional
TABLE 2: Fixed parameters of the WLAN/WiMAX multiband MSA.

\begin{tabular}{lc}
\hline Name & Value $(\mathrm{mm})$ \\
\hline$W_{1}$ & 44 \\
$W_{2}$ & 3 \\
$W_{3}$ & 0.5 \\
$W_{4}$ & 0.5 \\
$W_{5}$ & 22 \\
$L_{1}$ & 48 \\
$L_{2}$ & 10.5 \\
$L_{3}$ & 8 \\
$L_{9}$ & 12 \\
$H$ & 1.6 \\
\hline
\end{tabular}

PSO calling HFSS software algorithm, the efficiency of SFEPSO algorithm is improved by $95.7 \%$. The optimized size

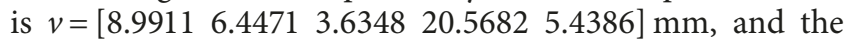
$\left|S_{11}\right|$ that satisfies the design specifications is shown in Figure 5.

Figure 6 shows the far-field pattern of the antenna at $2.45 \mathrm{GHz}, 3.5 \mathrm{GHz}$, and $5.5 \mathrm{GHz}$. It can be seen that the pattern of the E-plane of the antenna has an " 8 " shape and the pattern of the $\mathrm{H}$-plane is approximately circular. The antenna exhibits omnidirectional characteristics.

\section{Conclusion}

In optimal design of complex MSAs, we often combine global optimization algorithms such as particle swarm optimization (PSO) or genetic algorithm (GA) with full-wave electromagnetic simulation software such as HFSS, CST, or IE3D. The main disadvantage of the method is time-consuming. Aiming to solve the problem, the study proposes a self-renewing fitness prediction method based on PSO algorithm (SFEPSO) in which the fitness of next generation is predicted by the weighted average of the fitness of last two generations. The proposed method can avoid time-consuming fitness calculations and shorten optimization time. In order to ensure the accuracy and stability of the method 


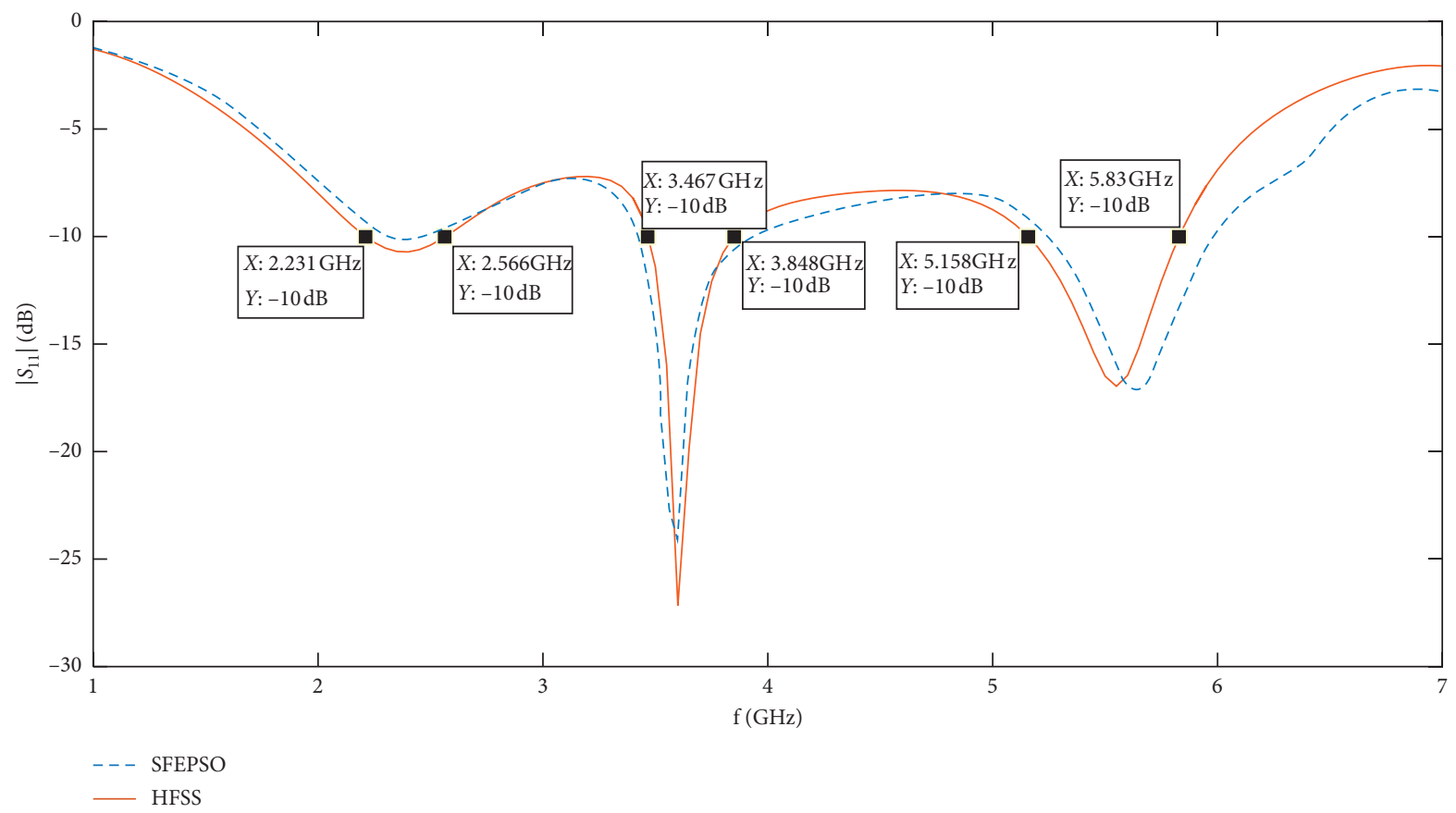

FIgURE 5: Results of the optimized WLAN/WiMAX multiband MSA.
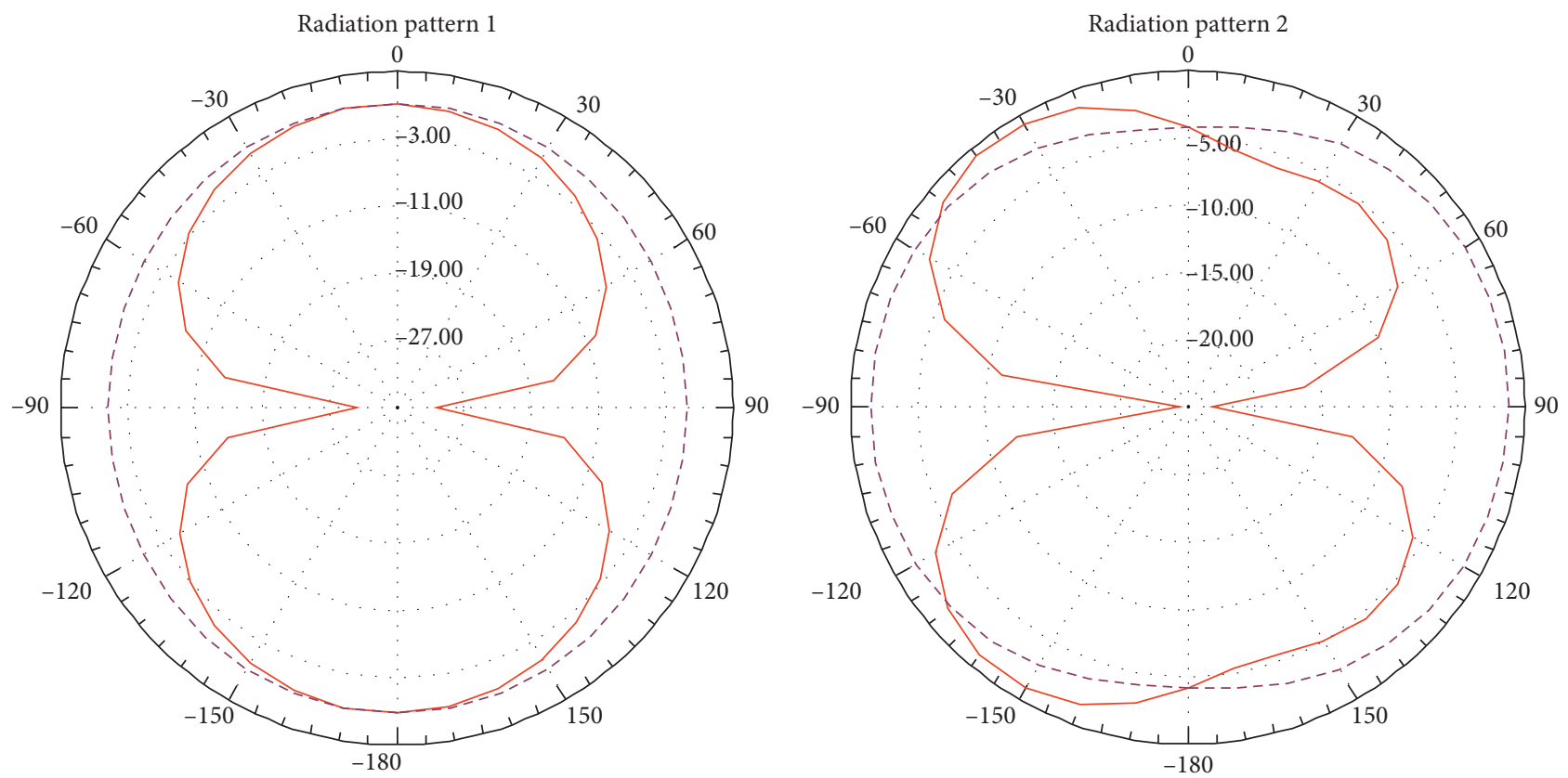

Curve info

Curve info

$$
\begin{aligned}
& \text { dB (Gain total) } \\
& \text { Setup 1: Last adaptive } \\
& \text { Freq = '2.45 GHz' Phi }=\text { '0 deg' } \\
& --- \text { dB (Gain total) } \\
& \text { Setup 1: Last adaptive } \\
& \text { Freq = '2 } 2.45 \mathrm{GHz} \text { Phi }=\text { '90 deg' }
\end{aligned}
$$

(a)

$$
\begin{aligned}
& -\mathrm{dB}(\text { Gain total }) \\
& \text { Setup 1: Last adaptive } \\
& \text { Freq = '3.5 GHz'Phi = '0 deg' } \\
& --- \text { dB (Gain total) } \\
& \text { Setup 1: Last adaptive } \\
& \text { Freq = '3.5 GHz' Phi = '90 deg' }
\end{aligned}
$$

(b)

FIGURE 6: Continued. 

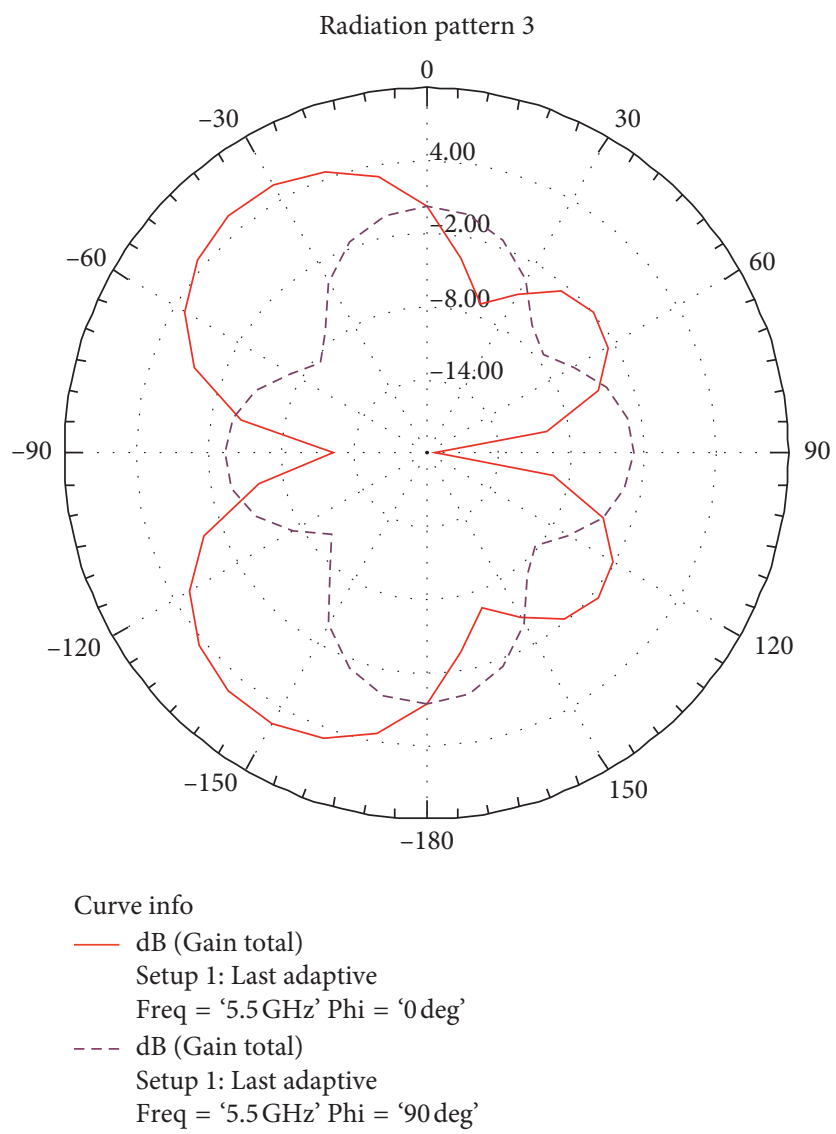

(c)

Figure 6: Radiation pattern of the optimized WLAN/WiMAX multiband MSA: (a) $2.45 \mathrm{GHz}$, (b) $3.5 \mathrm{GHz}$, and (c) $5.5 \mathrm{GHz}$.

and prevent particles from generating large errors as the number of iterations, the method is checked every several generations. In this paper, the SFEPSO algorithm is verified by designing an E-shaped dual-frequency MSA and a WLAN/WiMAX multiband MSA. The results show that the algorithm greatly reduces the number of times of calling fullwave electromagnetic simulation software and proves the effectiveness and accuracy.

\section{Data Availability}

The data used to support the findings of this study are included within the paper.

\section{Conflicts of Interest}

The authors declare that there are no conflicts of interest regarding the publication of this paper.

\section{Acknowledgments}

This work was supported by the National Natural Science Foundation of China (NSFC) under no. 61771225, the Key Research and Development Program Project of Social Development in Jiangsu Province under no. BE2016723, the Postgraduate Research \& Practice Innovation Program of
Jiangsu Province China under no. KYCX18_2326, and the Qinglan Project of Jiangsu Higher Education.

\section{References}

[1] D. M. Pozar, "Microstrip antennas," Proceedings of the IEEE, vol. 80, no. 1, pp. 79-91, 1992.

[2] B. Liu, H. Aliakbarian, Z. Ma, G. A. E. Vandenbosch, G. Gielen, and P. Excell, "An efficient method for antenna design optimization based on evolutionary computation and machine learning techniques," IEEE Transactions on Antennas and Propagation, vol. 62, no. 1, pp. 7-18, 2014.

[3] C. Gianfagna, H. Yu, M. Swaminathan, R. Pulugurtha, R. Tummala, and G. Antonini, "Machine-learning approach for design of nanomagnetic-based antennas," Journal of Electronic Materials, vol. 46, no. 8, pp. 4963-4975, 2017.

[4] X. H. Chen, X. X. Guo, J. M. Pei et al., "A hybrid algorithm of differential evolution and machine learning for electromagnetic structure optimization," in Proceedings of the 32nd Youth Academic Annual Conference of Chinese Association of Automation (YAC), Hefei, China, May, 2017.

[5] S. Sagiroglu and K. Güney, "Calculation of resonant frequency for an equilateral triangular microstrip antenna with the use of artificial neural networks," Microwave \& Optical Technology Letters, vol. 14, no. 2, pp. 89-93, 2015.

[6] K. S. R. Krishna, "Bandwidth and mutual coupling analysis of a circular microstrip MIMO antenna using artificial neural networks," Arabian Journal for Science \& Engineering, vol. 41, no. 8, pp. 3231-3238, 2016. 
[7] F. Chen and Y. Tian, "Modeling resonant frequency of rectangular microstrip antenna using CUDA-based artificial neural network trained by particle swarm optimization algorithm," The Applied Computational Electromagnetics Society Journal, vol. 29, no. 12, pp. 1025-1034, 2014.

[8] Y. B. Tian, S. L. Zhang, and J. Y. Li, "Modeling resonant frequency of microstrip antenna based on neural network ensemble," International Journal of Numerical Modelling: Electronic Networks, Devices and Fields, vol. 24, no. 1, pp. 78-88, 2011.

[9] Z. Zheng, X. Chen, and K. Huang, "Application of support vector machines to the antenna design," International Journal of RF and Microwave Computer-Aided Engineering, vol. 21, no. 1, pp. 85-90, 2015.

[10] W. Chen, P. Xing, and Q. Zou, "Detecting N6-methyladenosine sites from RNA transcriptomes using ensemble support vector machines," Scientific Reports, vol. 7, article 40242, 2017.

[11] S. Koziel and J. P. Jacobs, "Gaussian process antenna modeling using neighborhood-data-expanded training sets," in Proceedings of the 2013 IEEE Antennas and Propagation Society International Symposium (APSURSI), Orlando, FL, USA, July 2013.

[12] D. K. Neog, S. S. Pattnaik, D. C. Panda, S. Devi, B. Khuntia, and M. Dutta, "Design of a wideband microstrip antenna and the use of artificial neural networks in parameter calculation," IEEE Antennas and Propagation Magazine, vol. 47, no. 3, pp. 60-65, 2005.

[13] Y. Chen, Y.-B. Tian, Z. Qiang, and L. Xu, "Optimisation of reflection coefficient of microstrip antennas based on KBNN exploiting GPR model," IET Microwaves, Antennas \& Propagation, vol. 12, no. 4, pp. 602-606, 2018.

[14] C. Roy, T. Khan, and B. K. Kanaujia, "Performance parameters prediction of slotted microstrip antennas with modified ground plane using support vector machine," International Journal of Microwave and Wireless Technologies, vol. 9, no. 5, pp. 1169-1177, 2016.

[15] F. Y. Sun, Y. B. Tian, and Z. L. Ren, "Modeling the resonant frequency of compact microstrip antenna by the PSO-based SVM with the hybrid kernel function," International Journal of Numerical Modelling Electronic Networks Devices \& Fields, vol. 29, no. 6, pp. 1129-1139, 2016.

[16] J. P. D. Villiers and J. P. Jacobs, "Gaussian process modeling of CPW-FED slot antennas," Progress in Electromagnetics Research, PIER 98, vol. 98, no. 4, pp. 233-249, 2009.

[17] J. P. Jacobs and J. P. D. Villiers, "Gaussian-process-regressionbased design of ultrawide-band and dual-band CPW-FED slot antennas," Journal of Electromagnetic Waves and Applications, vol. 24, no. 13, p. 10, 2010.

[18] J. P. Jacobs, "Efficient resonant frequency modeling for dualband microstrip antennas by Gaussian process regression," IEEE Antennas and Wireless Propagation Letters, vol. 14, no. 3, pp. 337-341, 2015.

[19] R. E. Smith, B. A. Dike, and S. A. Stegmann, "Fitness inheritance in genetic algorithms," in Proceedings of the ACM 1995 Symposium on Applied Computing, DBLP, Nashville, TN, USA, February 1995.

[20] M. Salami and T. Hendtlass, "A fast evaluation strategy for evolutionary algorithms," Applied Soft Computing, vol. 2, no. 3, pp. 156-173, 2003.

[21] M. Reyes-Sierra and C. A. Coello, "Fitness inheritance in multi-objective particle swarm optimization," in Proceedings of the Swarm Intelligence Symposium, Pasadena, CA, USA, June 2005.
[22] Z. Cui, J. Zeng, and C. Sun, "A fast particle swarm optimization," International Journal of Innovative Computing Information \& Control, vol. 2, pp. 148-155, 2009.

[23] Z. Cui, X. Cai, and Z. Shi, "Using fitness landscape to improve the performance of particle swarm optimization," Journal of Computational and Theoretical Nanoscience, vol. 9, no. 2, pp. 258-265, 2012.

[24] C. Sun, J. Zeng, J. Pan, S. Xue, and Y. Jin, "A new fitness estimation strategy for particle swarm optimization," Information Sciences, vol. 221, pp. 355-370, 2013.

[25] A. Xiao, B. Wang, C. Sun et al., "Fitness estimation based particle swarm optimization algorithm for layout design of truss structures," Mathematical Problems in Engineering, vol. 2014, Article ID 671872, 11 pages, 2014.

[26] L. Lu, L. Yanchun, L. Tingting et al., "Boost particle swarm optimization with fitness estimation," Natural Computing, vol. 18, no. 2, pp. 229-247, 2019.

[27] J. Kennedy and R. Eberhart, "Particle swarm optimization," in Proceedings of the IEEE International Conference on Neural Networks (ICNN'95), vol. 4, pp. 1942-1948, Perth, WA, Australia, December 1995.

[28] X.-H. Fan, Y.-B. Tian, and Y. Zhao, "Optimal design of Yagi microstrip antenna based on particle swarm optimization with fitness estimation," in Proceedings of the 2018 Progress In Electromagnetics Research Symposium, pp. 653-660, PIERS, Toyama, Japan, August 2018.

[29] M. Sauter, "Wireless local area network (WLAN)," in From GSM to LTE-Advanced Pro and 5G: An Introduction to Mobile Networks and Mobile Broadband, John Wiley \& Sons, Ltd, Hoboken, NJ, USA, 3rd edition, 2017.

[30] A. Sharma, G. Das, and R. K. Gangwar, "Dual-band circularly polarized hybrid antenna for WLAN/WiMAX applications," Microwave and Optical Technology Letters, vol. 59, no. 10, pp. 2450-2457, 2017. 


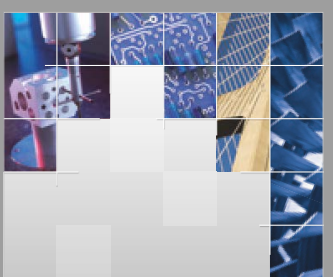

\section{Enfincering}
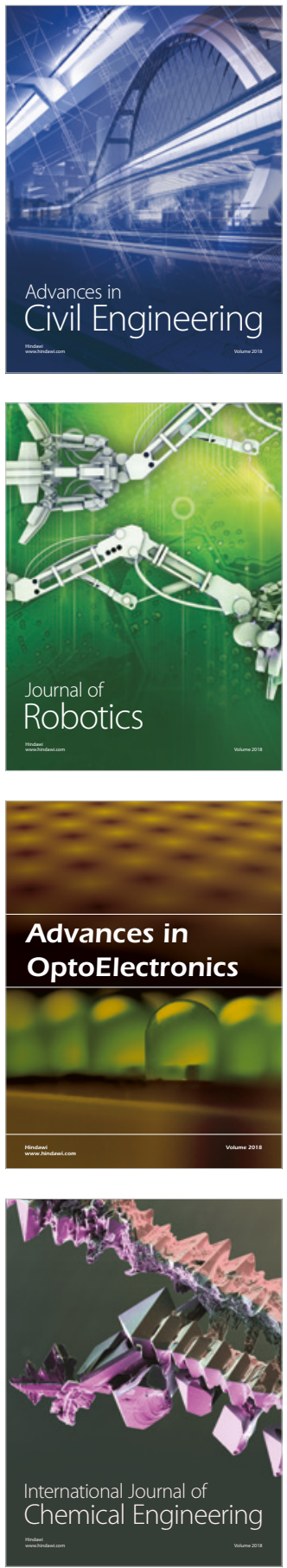

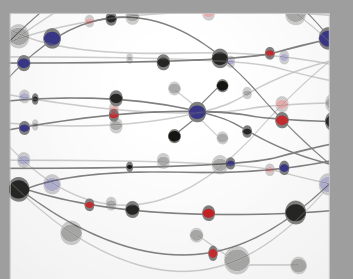

\section{Rotating \\ Machinery}

The Scientific World Journal

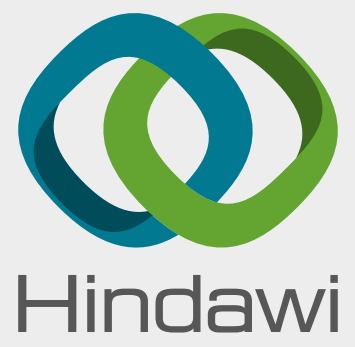

Submit your manuscripts at

www.hindawi.com
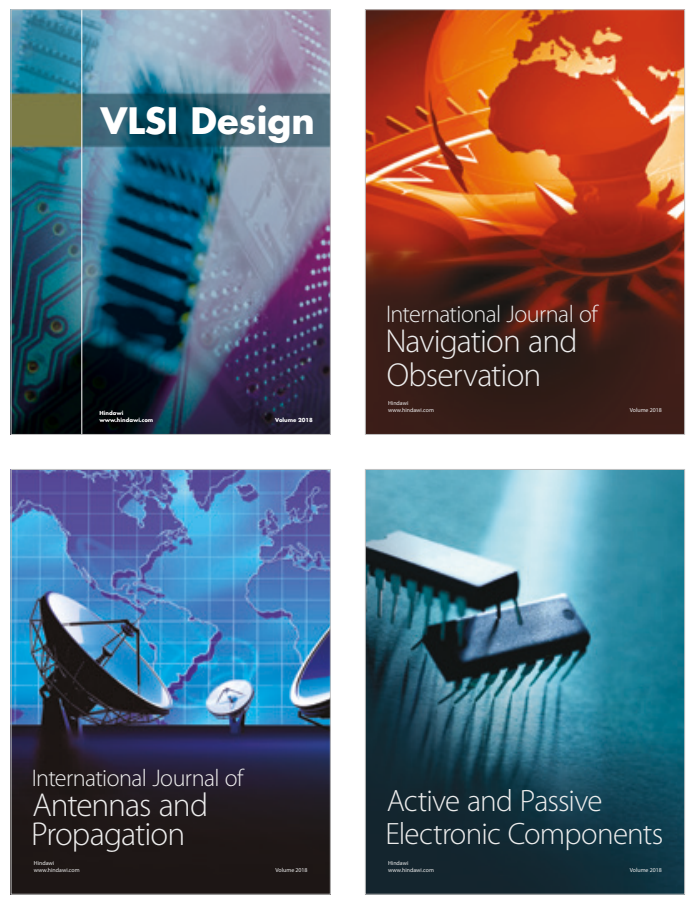
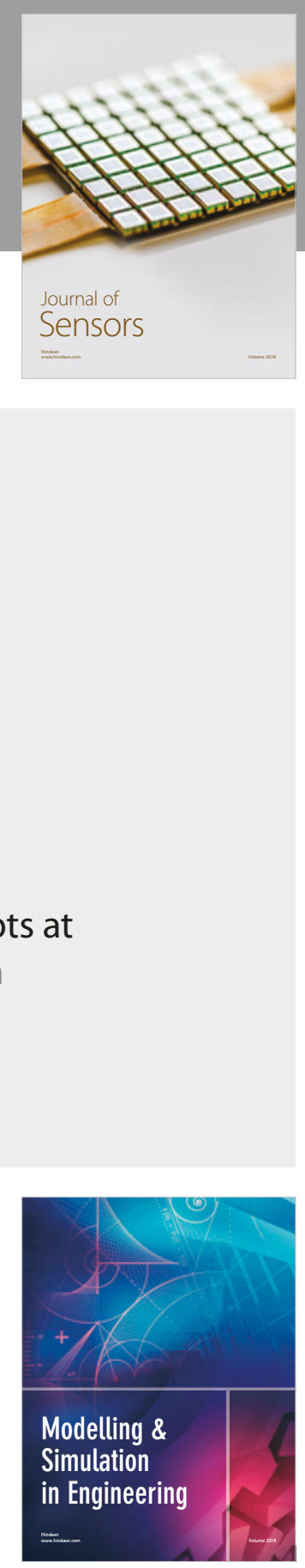

\section{Advances \\ Multimedia}
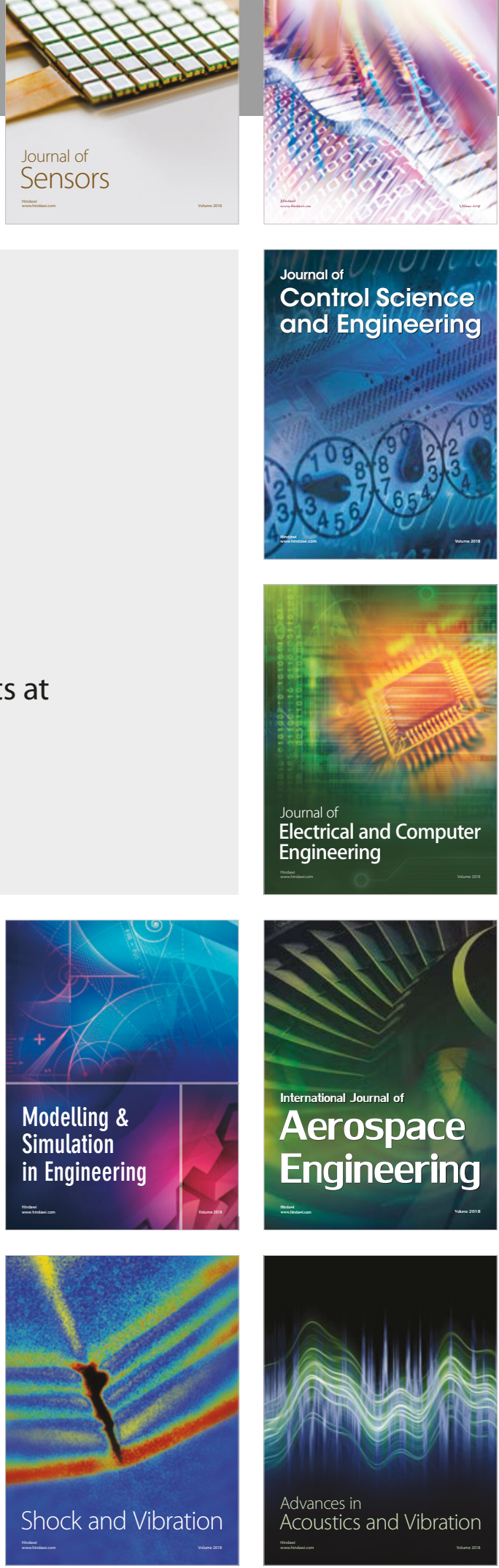